\title{
Precision studies of Drell-Yan transverse momentum distributions and the polarisation angular coefficients in Z-boson decays with the ATLAS detector
}

\author{
Aleksei Ezhilov* on behalf of the ATLAS Collaboration \\ National Research Centre "Kurchatov Institute" B.P.Konstantinov Petersburg Nuclear Physics \\ Institute, St. Petersburg, Russia \\ E-mail: alexey•ezhilovecern.ch \\ Oleg Fedin on behalf of the ATLAS Collaboration \\ National Research Centre "Kurchatov Institute" B.P.Konstantinov Petersburg Nuclear Physics \\ Institute, St. Petersburg, Russia \\ E-mail: oleg.fedin@cern.ch
}

\begin{abstract}
The ATLAS collaboration has performed precision measurements sensitive to the transverse momentum of the $Z / \gamma^{*}$ bosons, both directly through the transverse momentum of the dilepton pair and through the angular decorrelation as measured in the $\phi^{*}$ observable. These measurements are sensitive to soft resummation effects and hard jet emissions for small and large momentum transfers, respectively, probing QCD in a unique way. The studies carried out with $20.3 \mathrm{fb}^{-1}$ of data at a center-of-mass energy of $8 \mathrm{TeV}$ probe a wide dilepton invariant mass region from $12 \mathrm{GeV}$ to $150 \mathrm{GeV}$, both integrated and differential in the dilepton rapidity. The precision measurement of angular distributions of the Drell-Yan lepton pairs around the Z-boson mass peak provide a stringent test of the underlying QCD dynamics of the Z-boson production mechanisms through spin-correlation effects between initial and final state. A measurement is presented of the complete set of eight angular coefficients describing these distributions as determined from $20.3 \mathrm{fb}^{-1}$ of data collected with the ATLAS detector at a center-of-mass energy of $8 \mathrm{TeV}$ as a function of Z-boson rapidity and transverse momentum. The measurements are compared to precise theory calculations at NNLO in QCD and state-of-the-art MC generation at NLO in QCD. Strong discrimination power between different approaches of the QCD modeling and effects beyond NNLO are found.
\end{abstract}

XXIV International Workshop on Deep-Inelastic Scattering and Related Subjects

11-15 April, 2016

DESY Hamburg, Germany

${ }^{*}$ Speaker. 


\section{Introduction}

The Drell-Yan production of lepton pairs is a benchmark process at hadron colliders like the Large Hadron Collider (LHC). The production of Z-bosons with subsequent leptonic decays has both a clean and readily identifiable signature and a large event rate. It is a key process for precision measurements of electroweak (EW) parameters, and also allows to probe various aspects of the strong interaction, including parton distribution functions (PDFs), the strong coupling constant $\alpha_{s}$, and the behavior of processes involving multiple scales.

At all orders in QCD, the differential cross-section has a following general form:

$$
\begin{aligned}
\frac{\mathrm{d} \sigma}{\mathrm{d} p_{\mathrm{T}}^{z} \mathrm{~d} y_{z} \mathrm{~d} m_{z} \mathrm{~d} \cos \theta \mathrm{d} \phi} & =\frac{3}{16 \pi} \frac{\mathrm{d} \sigma^{U+L}}{\mathrm{~d} p_{\mathrm{T}}^{z} \mathrm{~d} y_{z} \mathrm{~d} m_{z}}\left\{\left(1+\cos ^{2} \theta\right)+\frac{1}{2} A_{0}\left(1-3 \cos ^{2} \theta\right)\right. \\
+A_{1} \sin 2 \theta & \cos \phi+\frac{1}{2} A_{2} \sin ^{2} \theta \cos 2 \phi+A_{3} \sin \theta \cos \phi+A_{4} \cos \theta \\
& \left.+A_{5} \sin ^{2} \theta \sin 2 \phi+A_{6} \sin 2 \theta \sin \phi+A_{7} \sin \theta \sin \phi\right\}
\end{aligned}
$$

where $p_{\mathrm{T}}$ and $y$ denote the transverse momentum and the rapidity of the $Z$-boson in its rest frame and the angles $\theta$ and $\phi$ are the polar and azimuthal decay angles, respectively, of the leptons in the gauge boson rest frame. The full five-dimensional differential cross-section is decomposed as a sum of nine harmonic polynomials, which depend on $\cos \theta$ and $\phi$, multiplied by corresponding helicity cross-sections that depend on the $Z$-boson transverse momentum, rapidity and invariant mass. The dimensionless angular coefficients $A_{0-7}\left(p_{\mathrm{T}}^{z}, y_{z}, m_{z}\right)$ represent ratios of helicity cross-sections with respect to the unpolarized one, $\sigma^{U+L}$.

This paper presents an inclusive measurement of the full set of eight $A_{i}$ coefficients and the distributions of transverse momentum $p_{\mathrm{T}}^{l l}$ and the angular variable $\phi^{*}$ (defined in equation 2.1) using charged lepton pairs (electrons or muons). These results are based on $20.3 \mathrm{fb}^{-1}$ of $p p$ collisions data collected at $\sqrt{s}=8 \mathrm{TeV}$ by the ATLAS experiment at the LHC. A detailed description of the ATLAS detector can be found in Ref. [1]. Both measurements allow probe perturbative QCD predictions at all orders of $\alpha_{s}$ complemented with Parton Showers(PS) and non-perturbative effects. The distributions of transverse momentum $p_{\mathrm{T}}^{l l}$ and the angular variable $\phi^{*}$ are also sensitive to soft-gluon resummation and hard jet emission.

\section{Measurements of the distributions of the transverse momentum and the angular variable $\phi^{*}$ distributions}

Measurements of $p_{\mathrm{T}}^{l l}$ require a precise understanding of the transverse momentum calibration and resolution of the final-state leptons. Associated systematic uncertainties affect the resolution in $p_{\mathrm{T}}^{l l}$ and limit the ultimate precision of the measurements, particularly in the low- $p_{\mathrm{T}}^{l l}$ domain [2]. To minimize the impact of these uncertainties, the $\phi_{\eta}^{*}$ observable was introduced as an alternative probe of $p_{\mathrm{T}}^{l l}$ :

$$
\phi_{\eta}^{*}=\tan \left(\frac{\pi-\Delta \phi}{2}\right) \sin \left(\theta_{\eta}^{*}\right)
$$



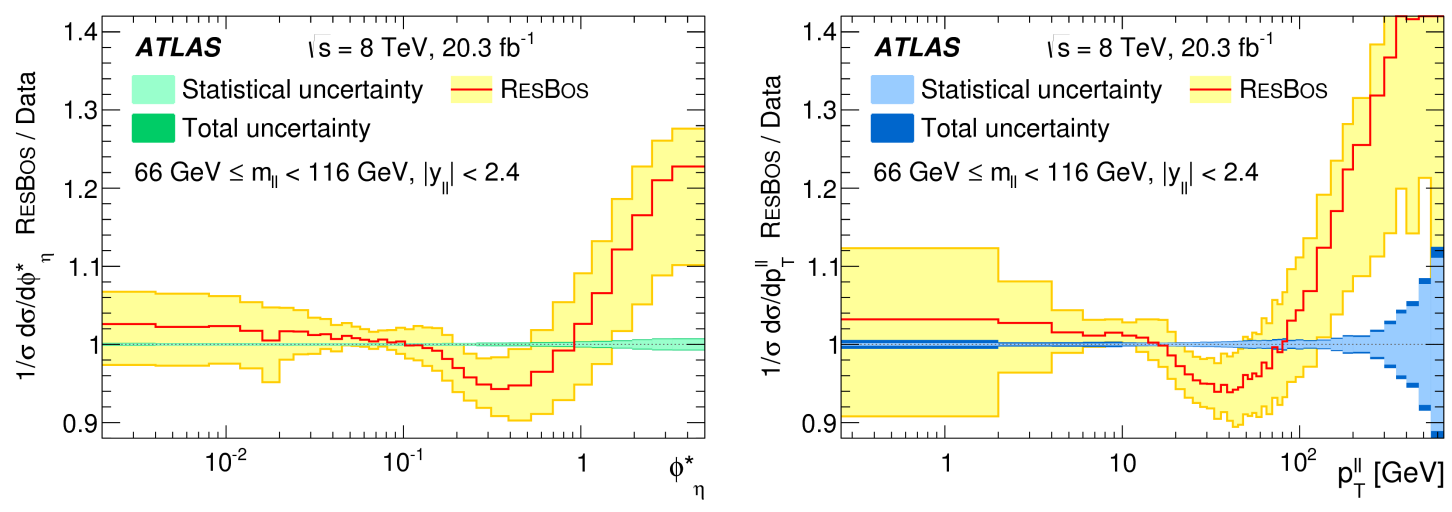

Figure 1: The ratio of the predictions of ResBos for the Z-boson mass peak and for $\left|y_{l l}\right|<2.4$ to the combined Born-level data for $(1 / \sigma) \mathrm{d} \sigma / \mathrm{d} \sigma \phi_{\eta}^{*}$ (left) and $(1 / \sigma) \mathrm{d} \sigma / \mathrm{d} \sigma p_{\mathrm{T}}^{l l}$ (right) [2].

where $\Delta \phi$ is the azimuthal angle in radians between the two leptons. The angle $\theta_{\eta}^{*}$ is a measure of the scattering angle of the leptons with respect to the proton beam direction in the rest frame of the dilepton system and is defined by $\cos \left(\theta_{\eta}^{*}\right)=\tanh \left[\left(\eta^{-}-\eta^{+}\right) / 2\right]$, where $\eta^{-}$and $\eta^{+}$are the pseudorapidities of the negatively and positively charged lepton, respectively. Therefore, $\phi_{\eta}^{*}$ depends exclusively on the directions of the two leptons, which are more precisely measured than their momenta.

A general comparison of the $\phi^{*}$ and $p_{\mathrm{T}}^{l l}$ distributions to the QCD predictions is provided by Figure 1. The scales on the abscissae in Figure 1 are aligned according to the approximate relation$\operatorname{ship} \sqrt{2} m_{z} \phi_{\eta}^{*} \approx p_{\mathrm{T}}^{l l}$.

At low values of $\phi_{\eta}^{*}$ and $p_{\mathrm{T}}^{l l}$, where non-perturbative effects and soft-gluon resummation are most important, the predictions from ResBos [3] are consistent with the data within the assigned theoretical uncertainties. However, at high values of of $\phi_{\eta}^{*}$ and $p_{\mathrm{T}}^{l l}$, which are more sensitive to the emission of hard partons, the predictions from ResBos don't describe the data.

Figure 2 (left) shows the ratio of $(1 / \sigma) \mathrm{d} \sigma / \mathrm{d} \sigma \phi_{\eta}^{*}$ predicted by various MC generators using different parton-shower approaches: POWHEG+PYTHIA (with both the AU2 and AZNLO tunes), PowheG+HERWIG and SHERPA to the data. For values of $p_{\mathrm{T}}^{l l}<50 \mathrm{GeV}$ for the $m_{l l}$ region around the Z-boson mass peak the best description is provided by PowHEG+PYTHIA (AZNLO), which was tuned to exactly this kinematic region in the $7 \mathrm{TeV}$ data. However, at high values of $p_{\mathrm{T}}^{l l}$ around the Z-boson mass peak and in other $m_{l l}$ regions this MC tune does not describe the data well and also does not outperform the Powheg+Pythia AU2 tune. The differences between SHERPA and the data are generally of a similar magnitude, but of opposite sign, to those seen for POWHEG+PYTHIA.

Figure 2 (right) shows the ratio of $\mathrm{d} \sigma / \mathrm{d} \sigma p_{\mathrm{T}}^{l l}$ as predicted by the fixed-order perturbative QCD predictions of DYNNLO [4] to Born-level data for six regions of $m_{l l}$. The predictions are not expected to describe the shape of the data for low values of $p_{\mathrm{T}}^{l l}$, where the effects of soft-gluon emissions are important. At $p_{\mathrm{T}}^{l l}>30 \mathrm{GeV}$ the shape of the $p_{\mathrm{T}}$ distribution is described within uncertainties by DYNNLO. However, the prediction is consistently low by about $15 \%$ compared to the data across all $m_{l l}$ ranges. A recent calculation suggests the size of order $\alpha_{s}^{3}$ corrections to be 

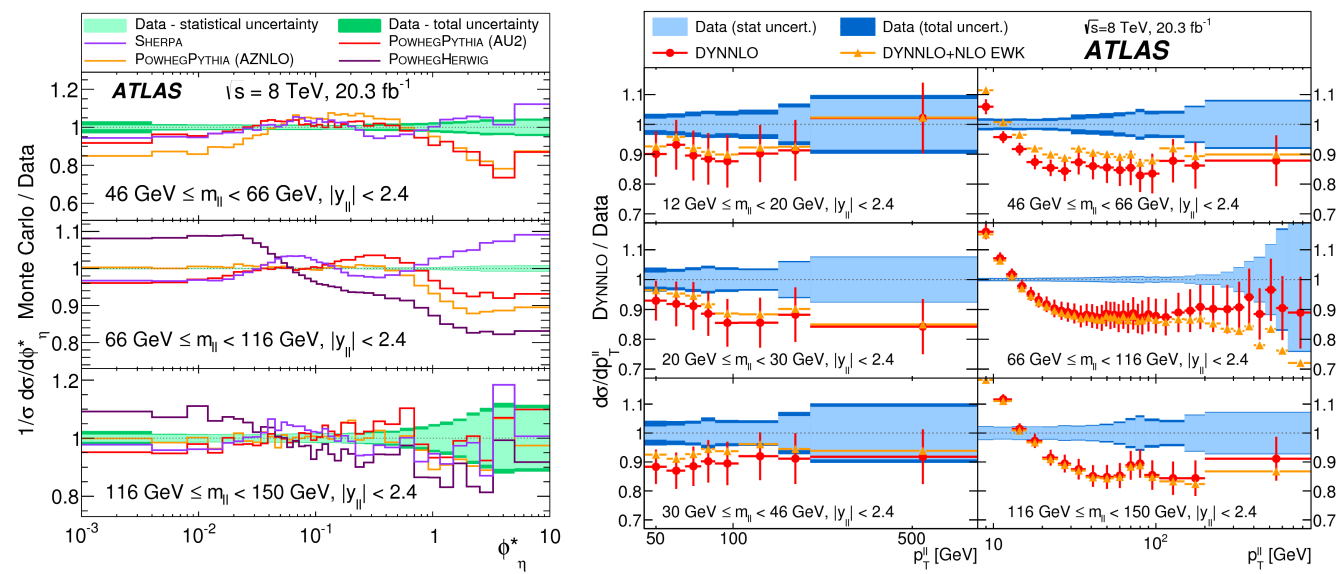

Figure 2: The ratio of $(1 / \sigma) \mathrm{d} \sigma / \mathrm{d} \sigma \phi_{\eta}^{*}$ predicted by various MC generators to the combined Born-level data, in three different regions of $m_{l l}$ for $\left|y_{l l}\right|<2.4$ (left) and the ratio of $\mathrm{d} \sigma / \mathrm{d} \sigma p_{\mathrm{T}}^{l l}$ as predicted by the DYNNLO MC generator to the combined Born-level data, for six different regions of $m_{l l}$. Two sets of DYNNLO predictions are shown, one of which includes NLO EW corrections and one does not (right) [2].

$+(5-10) \%$ for $p_{\mathrm{T}}^{l l}>60 \mathrm{GeV}$.

\section{Measurement of the angular coefficients in Z-boson events using lepton pairs}

The angular coefficients in Eq. 1.1 are not explicitly used as input to the theoretical calculations nor in the MC event generators. They can, however, be extracted from the shapes of the angular distributions with the moment method proposed in Ref. [5], owing to the orthogonality of the $P_{i}$ polynomials. The weighted average of the angular distributions with respect to any specific polynomial isolates an average reference value or moment of its corresponding coefficient. The moment of a polynomial $P(\cos \theta, \phi)$ over specific range of $p_{T}^{Z}, y_{Z}$ and $m_{Z}$ is defined by

$$
\langle P(\cos \theta, \phi)\rangle=\frac{\int P(\cos \theta, \phi) d \sigma(\cos \theta, \phi) d \cos \theta d \phi}{\int d \sigma(\cos \theta, \phi) d \cos \theta d \phi}
$$

The moment of each harmonic polynomial can be expressed as (see Eq. 1.1):

$$
\begin{aligned}
\left\langle\frac{1}{2}\left(1-3 \cos ^{2} \theta\right)\right\rangle & =\frac{3}{20}\left(A_{0}-\frac{2}{3}\right) ;\langle\sin 2 \theta \cos \phi\rangle=\frac{1}{5} A_{1} ;\left\langle\sin ^{2} \theta \cos 2 \phi\right\rangle=\frac{1}{10} A_{2} ;\langle\sin \theta \cos \phi\rangle=\frac{1}{4} A_{3} ; \\
\langle\cos \theta\rangle & =\frac{1}{4} A_{4} ;\left\langle\sin ^{2} \theta \sin 2 \phi\right\rangle=\frac{1}{5} A_{5} ;\langle\sin 2 \theta \sin \phi\rangle=\frac{1}{5} A_{6} ;\langle\sin \theta \sin \phi\rangle=\frac{1}{4} A_{7} .
\end{aligned}
$$

The moment method (3.1) relies on integration over the full phase space of the angular distributions, it cannot be applied directly to data, but is used to compute all the theoretical predictions.

The coefficients are extracted from the data by fitting templates of the $P_{i}$ polynomial terms, defined in Eq. 1.1, to the reconstructed angular distributions. Each template is normalized by free parameters for its corresponding coefficient $A_{i}$, as well by an additional common parameter representing the unpolarized cross-section. All these parameters are defined independently in each bin 
of $p_{\mathrm{T}}^{Z}$. To build the templates of the $P_{i}$ polynomials, the reference coefficients $A_{\text {ref }}$ for the signal MC sample are first calculated with the moments method. The information about the angular coefficients in the simulation is then available through the corresponding functional form of Eq. (1.1). Next, the MC event weights are divided by the value of this function on an event-by-event basis. When the MC events are weighted in this way, the angular distributions in the full phase space at the event generator level are flat. Effectively, all information about the Z-boson polarization is removed from the MC sample. The selection requirements, corrections, and event weights are then applied and finally nine separate template histograms are obtained after weighting by each of the $P_{i}$ terms. The templates $t_{i j}$ are thus three-dimensional distributions in the measured $\cos \theta, \phi$ and $p_{\mathrm{T}}^{l l}$ variables in the boson rest frame. The sum of all signal templates normalized by their reference coefficients and unpolarized cross-sections agrees exactly with the three-dimensional reconstructed distribution expected for signal MC events. Templates $T_{B}$ are also built for each of the multijet, top+electroweak, and non-fiducial Z-boson backgrounds.

The maximum likelihood fit is performed on the reconstructed data to determine the coefficients. The likelihood is the product of Poisson probabilities across all $N_{\text {bins }}$ bins and of auxiliary constraints for each nuisance parameter $\beta_{m}$ (experimental and theoretical uncertainties) and $\gamma^{n}$ (systematic uncertainties from the limited size of the MC samples):

$$
\mathscr{L}\left(A, \sigma, \theta \mid N_{\mathrm{obs}}\right)=\prod_{n}^{N_{\mathrm{bins}}}\left\{P\left(N_{\mathrm{obs}}^{n} \mid N_{\mathrm{exp}}^{n}(A, \sigma, \theta)\right) P\left(N_{\mathrm{eff}}^{n} \mid \gamma^{n} N_{\mathrm{eff}}^{n}\right)\right\} \times \prod_{m}^{M} G\left(0 \mid \beta^{m}, 1\right),
$$

where all signal and background templates are summed over with their respective normalizations, the expected events $N_{\text {exp }}^{n}$ in each bin $n$ can be written as:

$$
N_{\exp }^{n}(A, \sigma, \theta)=\left\{\sum_{j=1}^{23} \sigma_{j} \times L \times\left[t_{8 j}^{n}(\beta)+\sum_{i=0}^{7} A_{i j} \times t_{i j}^{n}(\beta)\right]+\sum_{B}^{\text {bkgs }} T_{B}^{n}(\beta)\right\} \times \gamma^{n},
$$

The theoretical fixed-order QCD predictions for Z-boson production at NLO and NNLO were obtained with DYNNLO. It was also compared with FEWZ at NNLO. The agreement between the two generators was found within uncertainties. The calculations from DYNNLO are shown at NNLO for $p_{\mathrm{T}}^{Z}>2.5 \mathrm{GeV} . A_{0}$ and $A_{2}$ are the fractions of transverse and longitudinal polarization.

The Lam-Tung relation predicts that $A_{0}-A_{2}=0$ at NLO, but it can be violated at higher orders. For $p_{\mathrm{T}}^{Z}>50 \mathrm{GeV}$ significant deviations from zero are observed, as shown on Fig. 3 (top left). The deviation is about a factor 2 larger than the predicted one. Since the impact of the PDF uncertainties on the calculations is very small, these deviations must be due to higher-order QCD effects.

Coefficients $A_{5,6,7}$ are equal to 0 at NLO and show some non-zero behavior at higher order of QCD mostly at high values of $p_{\mathrm{T}}^{Z}$. The results visible on the Fig.3 (top right and both bottom) are consistent with the predictions. However the calculations and the data are at the limit of the sensitivity. $A_{5,6,7}$ coefficients are measured for the first time and the additional tests provide an evidence of a non zero value with a significance of 3 standard deviations.

\section{Conclusions}

The results of precision measurements of the distributions of the transverse momentum and the angular variable $\phi^{*}$ distributions expand those presented by ATLAS at a center-of-mass energy 

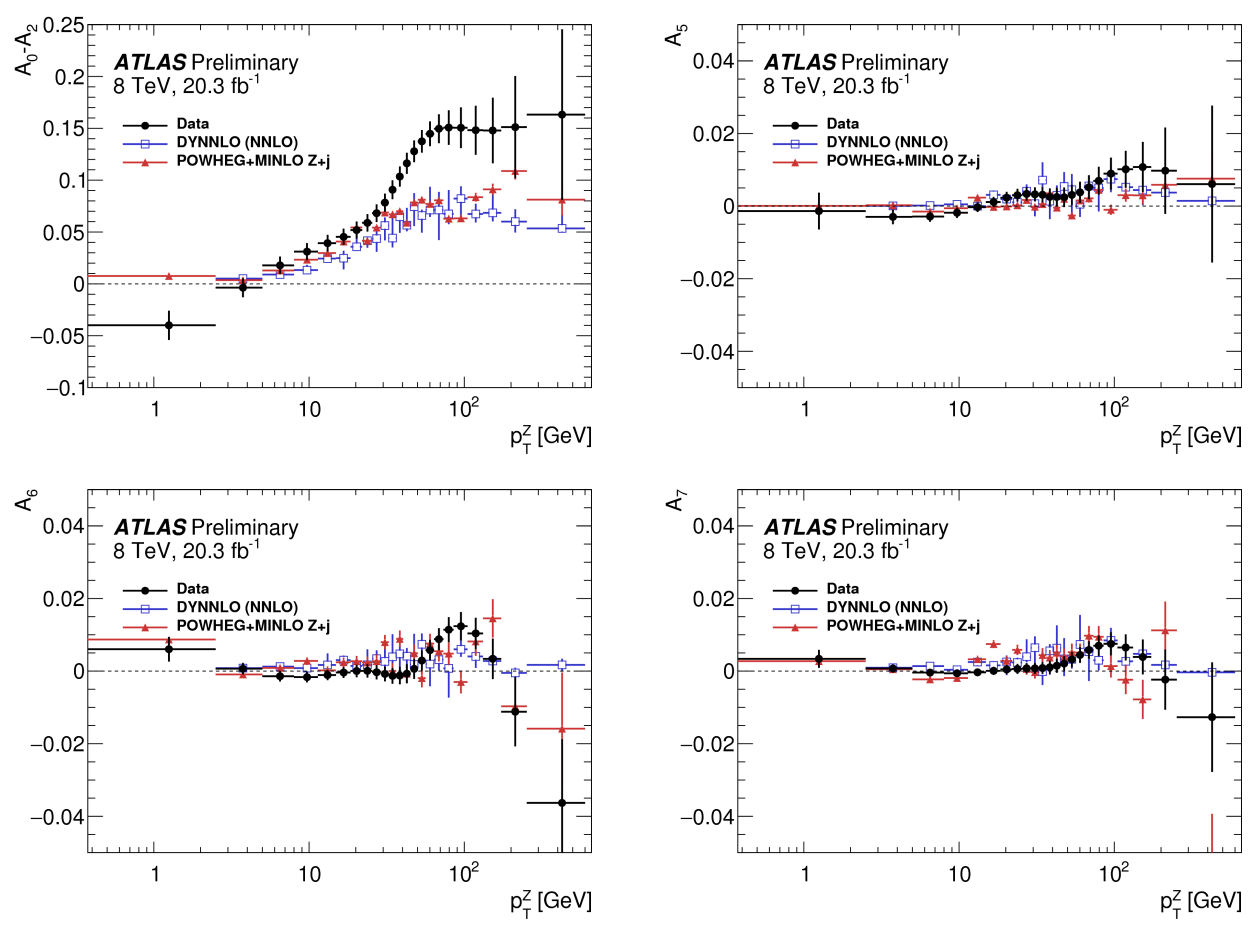

Figure 3: Distributions of the angular coefficients $A_{0}-A_{2}$ (top left), $A_{5}$ (top right) $A_{6}$ (bottom left) and $A_{7}$ (bottom right) as a function of $p_{\mathrm{T}}^{z}[6]$.

of $7 \mathrm{TeV}$. The measurements, compared to theoretical calculations and to predictions from MC generators, are precise enough to probe QCD corrections, complemented with Parton Showers(PS) and non-perturbative effects but are not precise enough to be sensitive to the inclusion of EW corrections. The results of angular coefficients measurements show a significant deviation observed for $A_{0}-A_{2}$ (Lam-Tung relation) from fixed-order prediction, indicating that higher-order QCD corrections are required to describe the data. The same result is observed for EW corrections at NNLO. Evidence at the $3 \sigma$ level is found for non-zero $A_{5,6,7}$ coefficients, consistent with the expectations from DYNNLO at $\mathrm{O}\left(\alpha_{s}^{2}\right)$.

\section{References}

[1] ATLAS Collaboration, 2008 JINST 3 S08003

[2] ATLAS Collaboration, arXiv:1512.02192

[3] C. Balazs, J.-W. Qiu and C. Yuan, Effects of QCD resummation on distributions of leptons from the decay of electroweak vector bosons, Phys. Lett. B355 (1995) 548-554

[4] S. Catani et al., Vector boson production at hadron colliders: A Fully exclusive QCD calculation at NNLO, Phys. Rev. Lett. 103 (2009) 082001

[5] E. Mirkes and J. Ohnemus, W and Z Polarization Effects in Hadronic Collisions, Phys. Rev. D 50 (1994) 5692, arXiv: 9406381 [hep-ph]

[6] ATLAS Collaboration, arXiv:1606.00689 